\title{
APPLICATION OF AN ENGINEERING INVISCID-BOUNDARY LAYER METHOD TO SLENDER THREE-DIMENSIONAL VEHICLE FOREBODIES
}

\author{
Christopher J. Riley* \\ NASA Langley Research Center, Hampton, VA
}

\begin{abstract}
$\underline{\text { Abstract }}$
An engineering inviscid-boundary layer method has been modified for application to slender threedimensional (3-D) forebodies which are characteristic of transatmospheric vehicles. An improved shock description in the nose region has been added to the inviscid technique which allows the calculation of a wider range of body geometries. The modified engineering method is applied to the perfect gas solution over a slender 3-D configuration at angle of attack. The method predicts surface pressures and laminar heating rates on the windward side of the vehicle that compare favorably with numerical solutions of the thin-layer Navier-Stokes equations. These improvements extend the 3 -D capabilities of the engineering method and significantly increase its design applications.
\end{abstract}

\section{Nomenclature}

$A, B, C, D, E$ circumferential conic section parameters $b, c, d \quad$ longitudinal conic section parameters

$e, f, g \quad$ shock curvature parameters

$\mathbf{e}_{\xi}, \mathbf{e}_{\beta} \quad$ unit vectors of shock-oriented coordinate system

$L \quad$ reference length

$M \quad$ Mach number

$n \quad$ coordinate normal to shock

$p \quad$ pressure

$q \quad$ heat transfer rate

$s \quad$ distance

$V \quad$ velocity magnitude

V velocity vector

$x, r, \phi \quad$ cylindrical coordinate system

$x, y, z \quad$ Cartesian coordinate system

$\alpha \quad$ angle of attack

*Aerospace Engineer, Aerothermodynamics Branch, Space Systems Division. Member AIAA.

Copyright (C)1993 by the American Institute of Aeronautics and Astronautics, Inc. No copyright is asserted in the United States under Title 17, U.S. Code. The U.S. Government has a royalty-free license to exercise all rights under the copyright claimed herein for Government purposes. All other rights are reserved by the copyright owner.

\begin{tabular}{ll}
$\kappa$ & shock curvature \\
$\nu$ & shock angle in a meridional plane \\
$\xi, \beta$ & shock coordinates \\
$\rho$ & density \\
$\Psi$ & stream function \\
Subscripts \\
\hline$b$ & body \\
$i$ & marching step \\
$k$ & meridional plane \\
$s u b$ & subsonic region \\
$w$ & wall \\
$\infty$ & freestream conditions
\end{tabular}

\section{Introduction}

The aerothermal design of hypersonic vehicles consists of evaluating vehicle geometries for a range of flow conditions. Numerical solutions of the Navier-Stokes (NS), parabolized Navier-Stokes (PNS), or viscous shocklayer (VSL) equations are usually cost-prohibitive except for the later stages of the design. For preliminary design and parametric studies, engineering inviscid-boundary layer methods are more appropriate. These methods predict reasonably accurate surface heating rates at a fraction of the computer cost of the NS algorithms.

An improved engineering inviscid-boundary layer method $^{1,2}$ has been developed by combining a simplified three-dimensional (3-D) inviscid technique ${ }^{3,4}$ with the axisymmetric analog concept ${ }^{5}$ and a set of approximate convective heating equations. ${ }^{6}$ This algorithm is referred to as THINBL (Three-dimensional Hypersonic INviscid-Boundary Layer method). The inviscid technique, which is similar to the thin shock-layer method of Maslen, ${ }^{7,8}$ improves the surface pressure distribution and extends the 3 -D capabilities of existing engineering methods. The method is applicable to perfect gas and equilibrium air flows at both laminar and turbulent conditions. Solutions have been obtained for axisymmetric shapes (paraboloids, sphere-cones) and simple 3 -D shapes (blunted elliptic cones) at angle of attack. ${ }^{1}$ Comparisons with experimental data and NS solutions have shown 


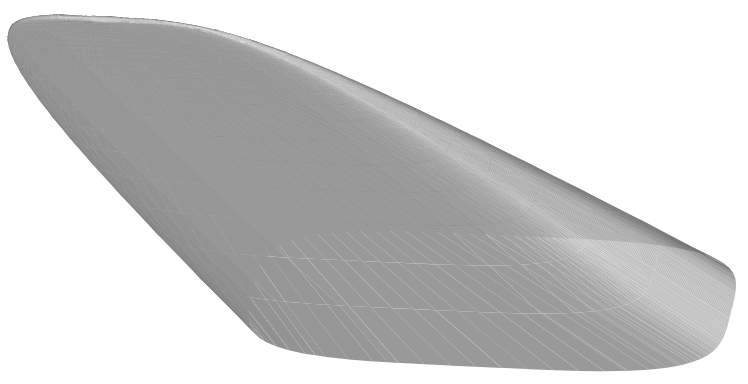

Figure 1. Slender 3-D forebody of a transatmospheric vehicle.

good agreement. This engineering method provides a unique capability for approximate flowfield solutions over 3 -D blunted noses as well as 3 -D afterbodies. ${ }^{1}$

The next step in the development of the THINBL code is to apply it to a realistic vehicle geometry. One such geometry is the slender 3 -D forebody characteristic of a transatmospheric vehicle (see Fig. 1). However, the first attempt at computing the flowfield over this body highlighted several limitations of the algorithm. The $3-\mathrm{D}$ inviscid technique used by THINBL is an inverse method: an assumed shock shape is iterated until the actual body shape is calculated. In the subsonic region near the nose, the possible shock surfaces assumed by THINBL cannot produce the slender $3-\mathrm{D}$ forebody shown in Fig. 1. For application to this configuration, the shock description requires modification. In addition, the sonic line location (which determines the subsonic boundary and the location where the solution can be marched downstream) varies greatly around the circumference of the body. This variation is currently neglected in the 3 -D inviscid technique. Also, due to the large curvature near the sides of the vehicle, some means of redistributing streamlines is necessary.

The purpose of this paper is to address these limitations and extend the capabilities of the engineering method THINBL to include more complex geometries such as the slender 3 -D forebody of a transatmospheric vehicle. This would significantly increase the design applications of engineering methods. Surface pressures and heating rates are computed for this geometry at angle of attack. Comparisons are made with NS solutions to demonstrate the accuracy of the present technique and to document the modifications.

\section{Analysis}

This section describes the engineering inviscidboundary layer method THINBL. The modifications

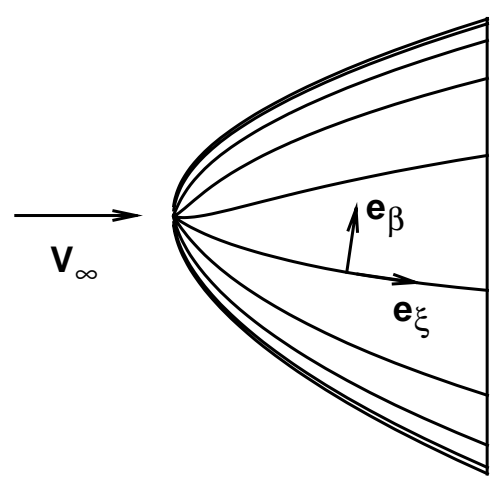

Figure 2. Shock-oriented curvilinear coordinate system: side view.

needed for the solution over slender hypersonic configurations are also discussed.

\section{$\underline{\text { THINBL }}$}

The engineering code THINBL (Three-dimensional Hypersonic INviscid-Boundary Layer method) is comprised of an approximate 3 -D inviscid method, a procedure for computing inviscid surface streamlines, and a set of approximate convective heating equations. The axisymmetric analog concept is employed for calculating the heating rates along surface streamlines. A complete description of THINBL is given in Refs. 1 and 2. A brief outline is presented here.

\section{Inviscid Method}

The 3 -D analysis is simplified by writing the inviscid equations in a shock-oriented curvilinear coordinate system as shown in Figs. 2 and 3 where $\xi$ and $\beta$ represent coordinates of a point on the shock surface and $n$ (not shown) is the inward distance normal to the shock. The unit vectors, $\mathbf{e}_{\xi}$ and $\mathbf{e}_{\beta}$, are tangent to the shock surface and are chosen such that $\mathbf{e}_{\xi}$ is in the direction of the tangential velocity just inside the shock surface. The unit vector $\mathbf{e}_{\beta}$ is then defined to be perpendicular to $\mathbf{e}_{\xi}$ and $\mathbf{e}_{n}$. A simplifying assumption is made that the velocity component in the $\mathbf{e}_{\beta}$ direction is equal to zero not only at the shock but throughout the shock layer. This approximation is similar to the axisymmetric analog used in the boundary-layer analysis and allows the solution along a given $\xi$-line to be computed independent of other lines. The momentum equations in the curvilinear coordinate system are then simplified by following the thin shock layer analysis of Maslen. ${ }^{7,} 8$ This yields explicit expressions for the pressure and normal velocity component along the $n$ coordinate as a function of a stream function $\Psi$. 


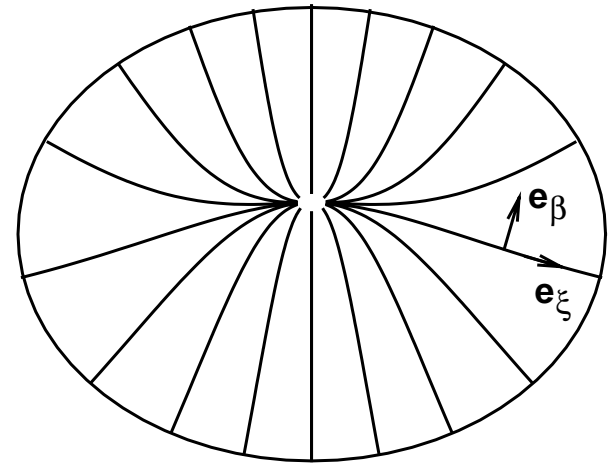

Figure 3. Shock-oriented curvilinear coordinate system: front view.

As stated previously, this technique is an inverse method in which the shock is assumed known. Flowfield properties are computed along $n$ from the known shock $\left(\Psi=\Psi_{s}\right)$ to $\Psi=0$ which defines the body. The shock shape is iterated until the computed body shape matches the actual body geometry. In the subsonic-transonic region, the shock is globally iterated until the correct body shape is obtained. Beyond this region, the shock surface is locally iterated as the solution is marched downstream along a given number of $\xi$-lines.

\section{Axisymmetric Analog}

The 3-D boundary-layer analysis is simplified by using the axisymmetric analog ${ }^{5}$ as is done in most engineering aerothermal methods. The 3 -D boundary-layer equations are first written in a inviscid surface streamline coordinate system. The crossflow velocity component tangent to the surface but normal to the streamline is then assumed to be equal to zero throughout the boundary layer. This simplification reduces the 3 -D boundary-layer equations to the axisymmetric form provided the distance along the streamline is substituted for the surface distance and the scale factor describing the divergence of the streamlines is interpreted as the axisymmetric body radius. Axisymmetric boundary-layer methods can then be coupled to the 3 - $\mathrm{D}$ inviscid technique.

Before applying the axisymmetric analog, inviscid surface streamlines are computed from the approximate inviscid solution. These body streamlines may be calculated from the surface pressure distribution ${ }^{9}$ or from the velocity components. ${ }^{10,13}$ The approximate inviscid method used in THINBL predicts accurate surface pressures, but the direction of the velocity on the surface is not accurate due to the assumption of zero velocity in the $\mathbf{e}_{\beta}$ direction. Therefore, streamlines are calculated from the surface pressures in THINBL. The equations for computing the streamlines are given in Refs. 1 and 2 .
Although the surface streamlines can be determined after the inviscid solution has already been calculated, it is more convenient to compute the inviscid solution and the streamlines simultaneously. In THINBL, streamline information (such as its orientation and the scale factor describing the divergence of streamlines) is integrated along a $\xi$-line along with the inviscid flowfield variables.

\section{Boundary-Layer Method}

The axisymmetric analog allows any axisymmetric boundary-layer method to be applied along an inviscid surface streamline. In THINBL, a set of approximate convective-heating equations developed by Zoby ${ }^{6}$ is used for the boundary-layer solution. Laminar and turbulent heating rates may be calculated from these relations for both perfect gas and equilibrium-air flows. Approximate expressions for the boundary-layer thickness at both laminar and turbulent conditions are also given in Ref. 6 . Boundary-layer edge conditions are found by interpolating in the approximate inviscid solution a distance from the wall equal to the boundary-layer thickness. This approach has been demonstrated to approximately account for the effects of entropy-layer swallowing.

\section{Modifications}

To compute the flow field over the slender configuration in Fig. 1, several areas of THINBL require modification. Changes in the shock shape near the nose; the handling of the subsonic region; and the distribution of the $\xi$-lines around the body are discussed.

\section{Shock Shape}

In the subsonic region surrounding the nose of the vehicle, the shock surface must be globally iterated. Presently, the shock surface is described by three longitudinal conic sections with the circumferential variation given by an ellipse. In a meridional plane, the longitudinal fit is given by the conic section

$$
\left(r^{2}+b x^{2}-2 c x+2 d x r\right)_{k}=0
$$

where $k$ represents the local meridional plane and the parameters $(b, c, d)_{k}$ govern the shape of the conic. The cylindrical coordinates $(x, \phi, r)$ are defined in a wind-oriented system with the $x$ axis aligned with the freestream velocity. Since one ellipse is used for the circumferential variation of the shock, only three meridional planes $(\phi=0,90,180 \mathrm{deg})$ are needed to completely describe the shock. The ellipse is given by

$$
r^{2}\left(A \cos ^{2} \phi+\sin ^{2} \phi\right)+r C \cos \phi=E
$$

where $A, C, E$ depend on the local values of $r_{k}$ from the three meridional planes and are functions of $x$. This form 
of the shock produces a body that can similarly be described by conic sections with an elliptical cross-section. However, the cross-section of the slender 3 -D forebody shown in Fig. 1 cannot be described by a single ellipse nor can the body be accurately fitted with a conic section in the planform view. Changes are required in both the axial and the cross-sectional fits of the shock surface for application to this vehicle.

A more general axial fit for the shock is the specification of the longitudinal shock curvature with a quadratic in $x$ as

$$
\kappa_{k}=\left(e+f x+g x^{2}\right)_{k}
$$

where $\kappa$ is the curvature. The radius is computed by an integration of

$$
\frac{d \sin \nu_{k}}{d x}=-\kappa_{k} \quad \frac{d r_{k}}{d x}=\tan \nu_{k}
$$

where $\tan \nu$ represents the shock slope. This fit was suggested by Maslen ${ }^{11}$ and produces conic as well as nonconic body shapes. The cross-sectional fit is improved by matching conic arcs. Each conic arc is described by

$r^{2}\left(A \cos ^{2} \phi+B \sin \phi \cos \phi+\sin ^{2} \phi\right)+r(C \cos \phi+D \sin \phi)=E$

At the match points between the arcs, the radius and its first and second derivatives with respect to $\phi$ are forced to be continuous. Cheatwood and DeJarnette ${ }^{12}$ follow a similar approach in curve-fitting body geometries except that no constraint is placed on the second derivative of the radius. The addition of conic arcs increases the number of parameters that describe the shock and allows for the calculation of body geometries with non-elliptical crosssections.

\section{Subsonic Region}

The global iteration of the shock surface in the subsonic region involves matching the body shape computed from the approximate flowfield equations to the actual body geometry. To insure a good starting solution for the downstream marching procedure, the matched body points are located at the end of the subsonic region where the flow on all $\xi$-lines is supersonic. For simplicity, the end of the subsonic region in the THINBL code is assumed to be located at $x=x_{s u b}$ where $x_{s u b}$ is constant and encompasses the entire subsonic region. The variation of $x_{s u b}$ with $\phi$ is neglected for the previous applications to a blunted elliptic cone at angle of attack.

However, the end of the subsonic region is not as conveniently defined for the case of a slender 3-D forebody. The flow near the "edges" of the configuration remains subsonic at much longer distances than the flow near the lower and upper symmetry planes. It is impractical to globally iterate the shock surface over this entire region with only a few parameters. For this reason, $x_{s u b}$ is now allowed to vary for each of the meridional planes used in the shock fit.

\section{$\xi$-Line Distribution}

One of the difficulties in tracing $\xi$-lines is that they tend to wrap around the 3 -D shock surface. A uniform distribution of $\xi$-lines is not guaranteed. For the previous applications to axisymmetric shapes and blunted elliptic cones at angle of attack, this problem is handled by grouping the $\xi$-lines in the stagnation region such that an even distribution is obtained at some point downstream. However, for a slender 3-D forebody, the flow is highly three-dimensional near the edges of the configuration and $\xi$-lines diverge rapidly from this region.

To insure a uniform distribution of $\xi$-lines as the solution is marched downstream, $\xi$-lines are redistributed at every axial station. Inviscid flowfield and streamline variables are integrated along a number of lines from $x_{i}$ to $x_{i+1}$. A new set of $\xi$-lines is chosen at $x_{i+1}$ that uniformly encompasses the shock. Flowfield and streamline variables associated with the new set of $\xi$-lines are found by interpolation between the previous lines. The process is repeated at each subsequent marching step. This approach has been suggested and used by Hamilton ${ }^{13}$ to compute the streamlines and surface heating over a complex vehicle.

\section{Results and Discussion}

Surface pressure and heating rate distributions are presented at laminar, perfect gas conditions over an axisymmetric power-law body and a slender 3-D forebody at angle of attack to assess the improvements and accuracy of the THINBL code. All variables are nondimensionalized as follows: pressure by $\rho_{\infty} V_{\infty}^{2}$, heating rate by $\rho_{\infty} V_{\infty}^{3}$, and distances by a reference length $L$.

\section{Axisymmetric Power-Law Body}

To examine the accuracy of the longitudinal shock fit for non-conic body shapes, the flowfield is computed over an axisymmetric power-law geometry given by

$$
r_{b}=x_{b}^{1 / 4}
$$

This geometry models the planform view of the slender $3-\mathrm{D}$ configuration in Fig. 1, and the configuration cannot be described adequately by a conic section. The freestream conditions are $M_{\infty}=15, \rho_{\infty}=0.00949$ $\mathrm{kg} / \mathrm{m}^{3}$, and $T_{\infty}=234 \mathrm{~K}$. The wall temperature is $T_{w}=1256 \mathrm{~K}$ and the reference length is $L=0.10 \mathrm{~m}$. Figure 4 gives the body shape calculated by the conic section shock, and Fig. 5 shows the body geometry which is predicted by assuming that the shock curvature is given by a quadratic in $x$. Excellent agreement between the computed and actual body geometries is given by the modified shock shape. The shock curvature predicted by both longitudinal fits is presented in Fig. 6. The quadratic fit allows for a much smoother transition between the subsonic 


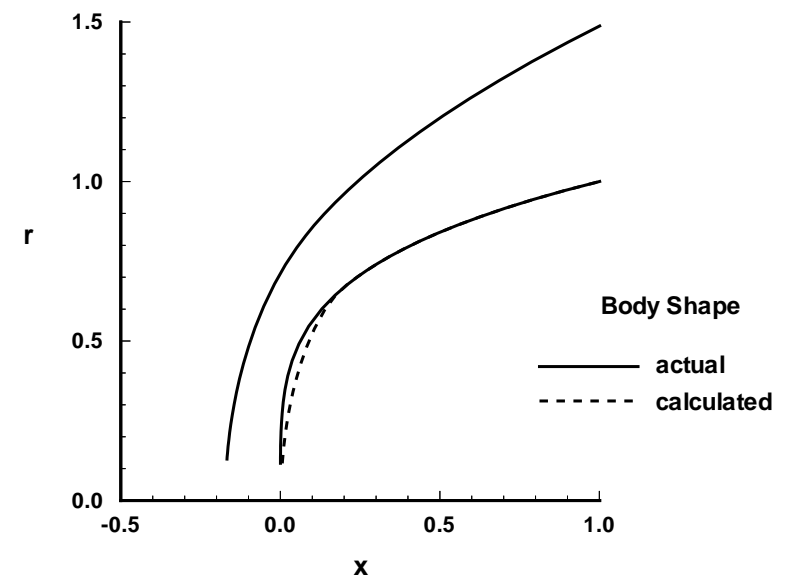

Figure 4. Comparison of body shapes produced by conic section shock.

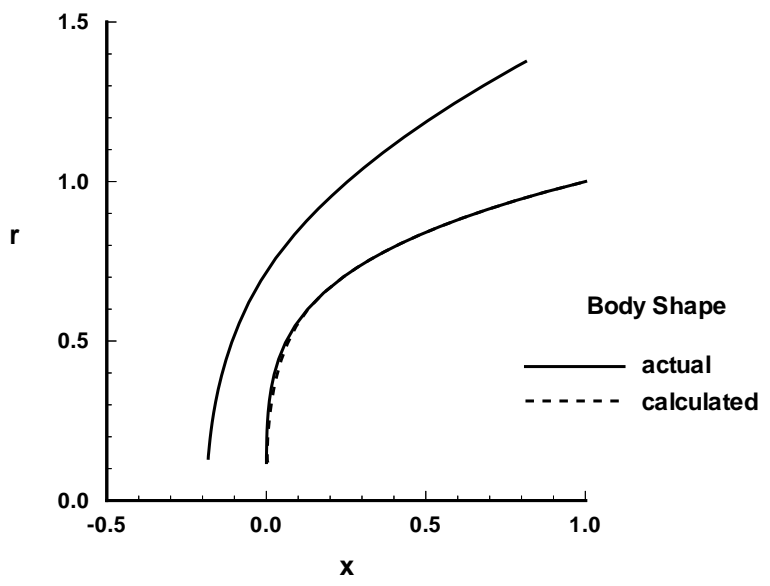

Figure 5. Comparison of body shapes produced by quadratic for shock curvature.

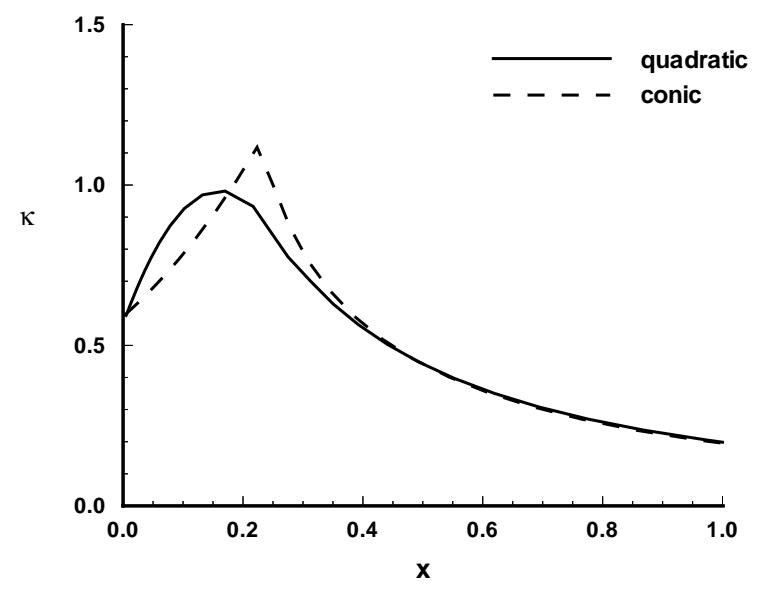

Figure 6. Shock curvature distribution.

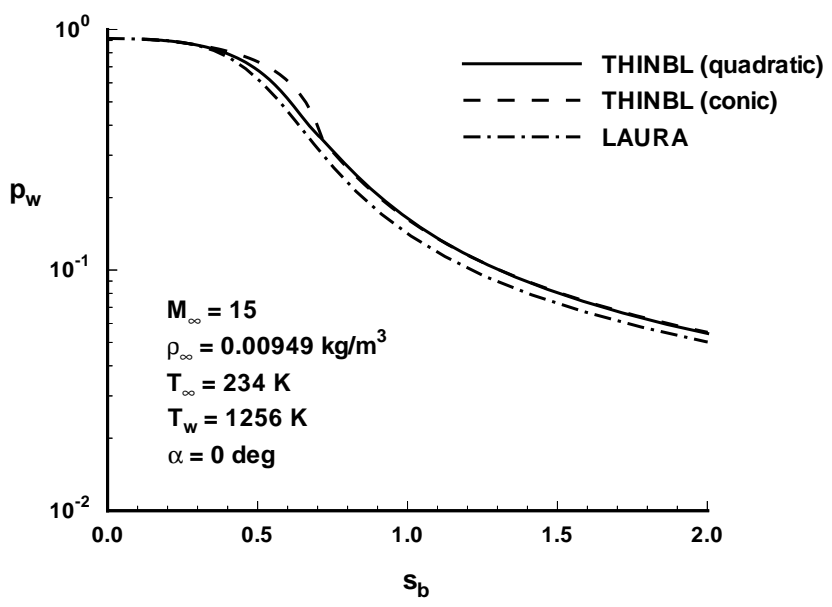

Figure 7. Surface pressure comparison for axisymmetric power-law body.

region $(x<0.25)$ and the supersonic region downstream.

Nondimensional surface pressures and heating rates are shown in Figs. 7 and 8 for the axisymmetric powerlaw geometry. The distance $s_{b}$ is the distance from the origin and is given by

$$
s_{b}=\left[x_{b}^{2}+r_{b}^{2}\right]^{1 / 2}
$$

Near the nose, $s_{b}$ approximates the distance along the surface. Results of THINBL with the quadratic and conic shock shapes are compared with a solution given by the thin layer NS algorithm LAURA. ${ }^{14}$ Because of the error between the calculated and actual body shapes, THINBL with the conic shock shape overpredicts the pressures generated by LAURA by as much as 35 percent. The modified version that uses the quadratic for the shock curvature gives more accurate (within 10 percent) and smoother pressures in the nose region. Note that both versions predict the same pressures downstream $\left(s_{b}>0.75\right)$. In Fig. 8 , the surface heating rates predicted by the modified THINBL follow the same trends as the heating rates from LAURA. The heating increases away from the stagnation point due to the shock layer becoming thinner. The original version of THINBL does not account for this effect.

\section{Slender 3-D Forebody}

To examine the accuracy of the improved $3-\mathrm{D}$ shock description, the flowfield is computed over a slender 3-D forebody at $10 \mathrm{deg}$ angle of attack. This geometry is characteristic of a transatmospheric vehicle. The side view is described by a circular nose followed by a line inclined at 5 deg; the planform is described by a power law; and the cross-section is given by a super-ellipse where the $\cos ^{2} \phi$ and $\sin ^{2} \phi$ terms in Eq. 2 have been replaced by $\cos ^{4} \phi$ and $\sin ^{4} \phi$. All three views of the vehicle are 


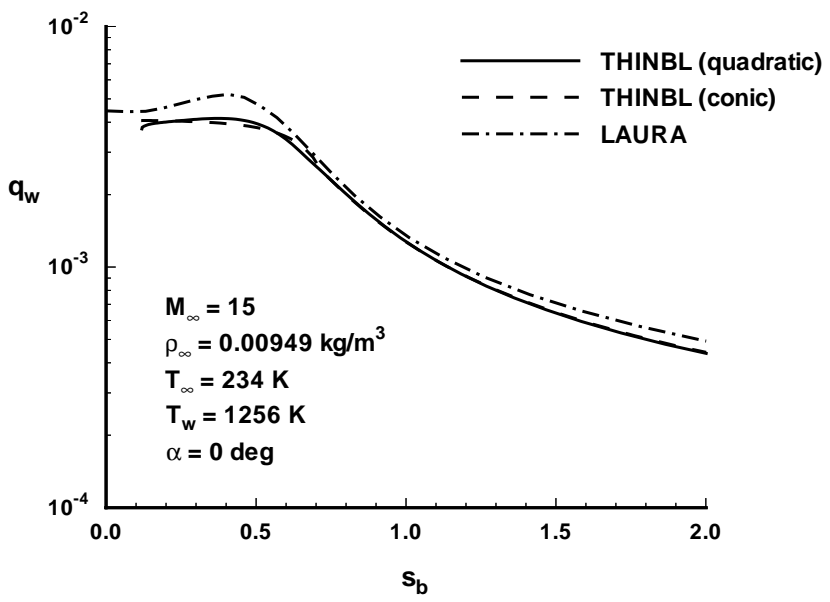

Figure 8. Surface heating comparison for axisymmetric power-law body.

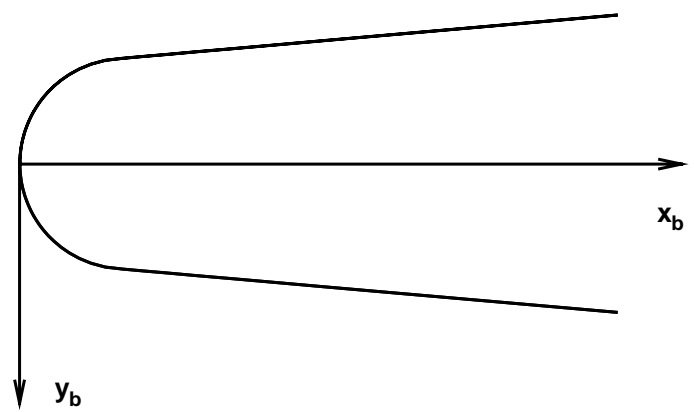

Figure 9. Slender 3-D forebody: side view.

shown in Figs. 9 - 11. Note that the three views are not drawn to the same scale. The freestream conditions are $M_{\infty}=10, \rho_{\infty}=0.00170 \mathrm{~kg} / \mathrm{m}^{3}$, and $T_{\infty}=48.3 \mathrm{~K}$. The wall temperature is $T_{w}=288 \mathrm{~K}$ and the reference length is $L=1.905 x 10^{-4} \mathrm{~m}$. Surface pressures and heating rates generated by THINBL are compared with thin layer NS results from the LAURA algorithm. Ninety-one $\xi$-lines are used in THINBL. They are distributed only on the lower half of the vehicle because of the code's inability to handle the separation present on the leeside. The $\xi$-lines are redistributed at each axial location to control their divergence from the sides of the vehicle. A grid of 40 cells in the axial direction, 200 cells around the circumference of the body, and 64 cells in the normal direction is used to obtain the LAURA solution. Both the $\xi$-lines in THINBL and the circumferential cells in LAURA are clustered in the high curvature regions near $y_{b}=0$ (See Fig. 11). THINBL requires 15 minutes to obtain a solution on a Sun Sparcstation while the LAURA solution required 25 hours on a Cray Y-MP supercomputer.

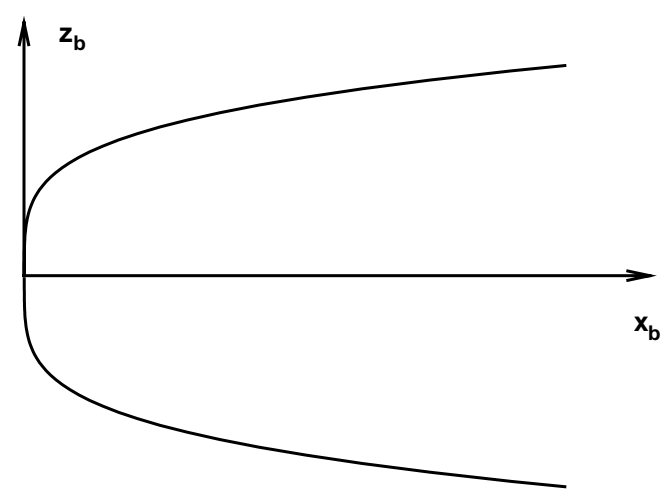

Figure 10. Slender 3-D forebody: top view.

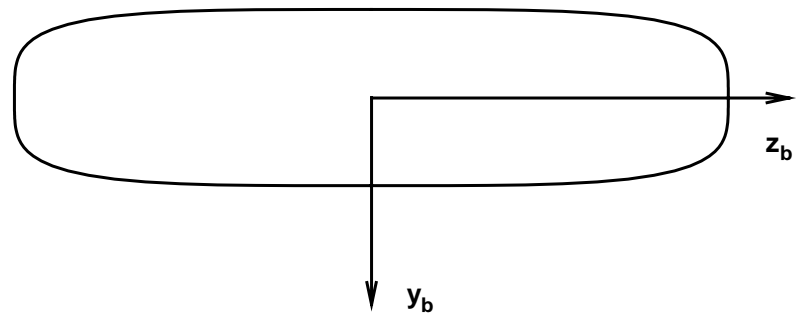

Figure 11. Slender 3-D forebody: rear view.

Axial surface pressures and heating rates are shown in Figs. 12 and 13 for the windward plane of symmetry. The surface pressures compare well with the pressures from the LAURA solution on the nose, but underpredict the pressures aft of the nose by 25 percent. Similar discrepancies have been seen downstream of the sphere-cone juncture of blunted slender cones. ${ }^{4}$ One of the thin shocklayer assumptions in THINBL's inviscid technique is that the local shock and body slopes are approximately equal. In the region near the nose of the slender 3 -D forebody and near the sphere-cone juncture of a blunted slender cone, this assumption is inaccurate. As a result, the surface pressures are in error in this region. However, for a blunted slender cone, the errors diminish after a few nose radii as shown for a 5 deg blunted cone in Fig. 14. Over the slender 3-D forebody, the differences between the THINBL and LAURA pressures persist for the 15 nose radii presented in Fig. 12. Because the lower surface of this $3-\mathrm{D}$ configuration is flat, the centerline flowfield is almost two-dimensional (2-D). The shock layer is thicker in a 2-D flow than in an axisymmetric flow. As a result, 


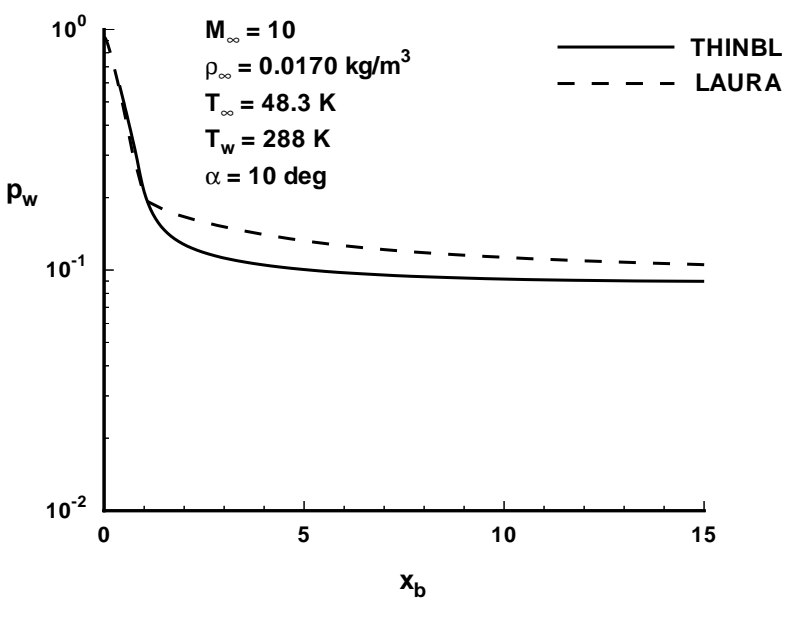

Figure 12. Axial pressure comparison along windward centerline of slender 3-D forebody.

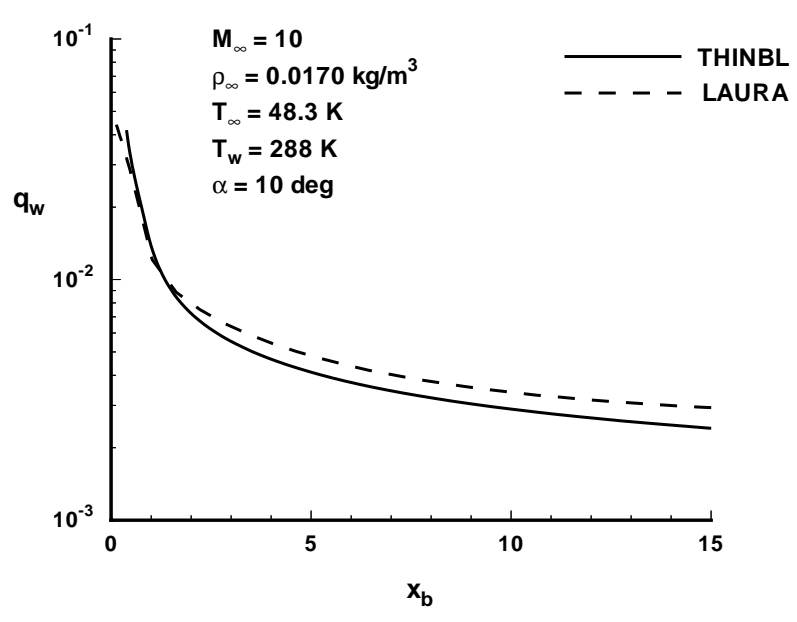

Figure 13. Axial heat transfer comparison along windward centerline of slender $3-D$ forebody.

the accuracy of the thin shock-layer assumptions is worse for a blunted slender wedge than for a cone. However, the approximations improve downstream as depicted in Fig. 15 for the inviscid flow over a 5 deg blunted wedge. It is felt that the comparison of centerline pressures over the slender 3-D vehicle will improve downstream as well.

Axial surface heating rates given by THINBL compare favorably (within 15 percent) with the LAURA solution in Fig. 13. Like the pressure comparison, the heating rates from THINBL underpredict the LAURA results downstream of the nose. These comparisons should again improve farther downstream. Preliminary comparisons with LAURA pressures and heating rates in the windward symmetry plane farther downstream confirm this hypothesis.

Circumferential pressures and heating rates are presented in Figs. 16 and 17 for an axial location of $x=10$. The surface variables are shown as a function of $z_{b}$ where $z_{b}=0$ represents the upper or lower symmetry plane

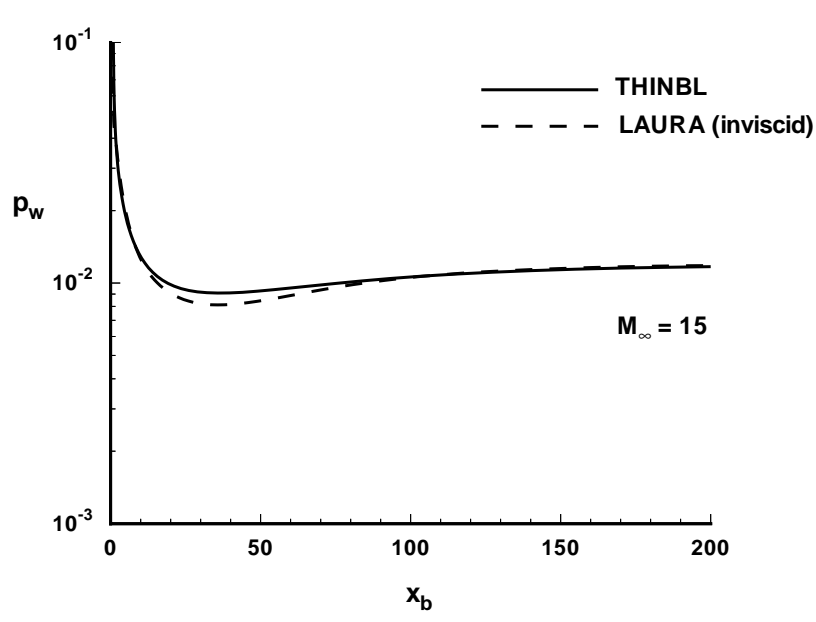

Figure 14. Axial pressure comparison for 5 deg blunted cone.

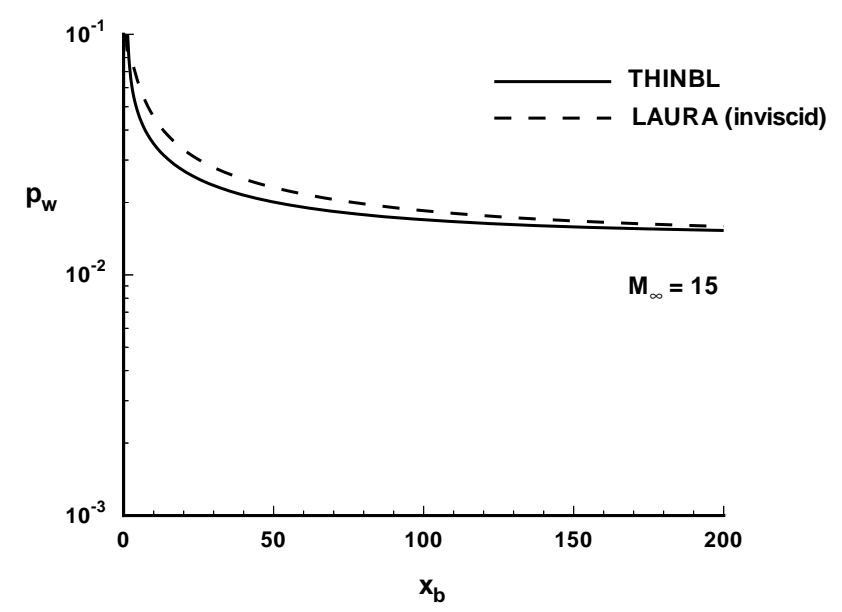

Figure 15. Axial pressure comparison for 5 deg blunted wedge.

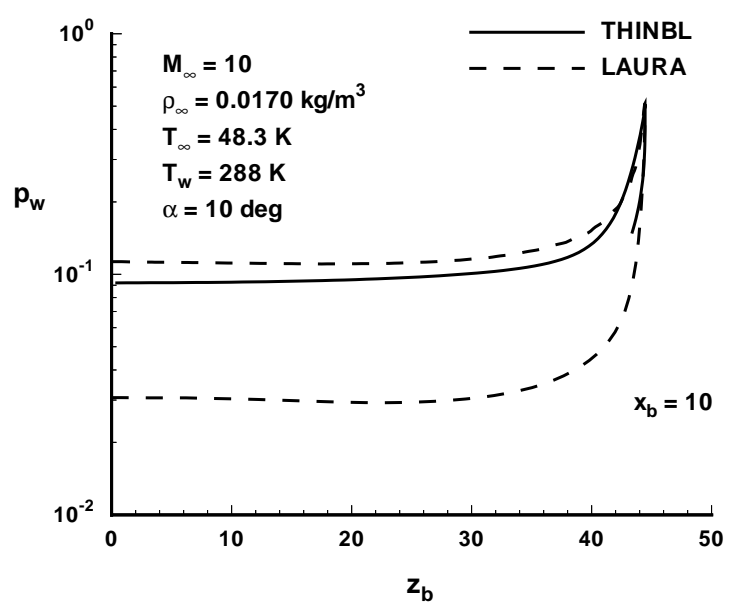

Figure 16. Circumferential pressure comparison for slender 3-D forebody. 


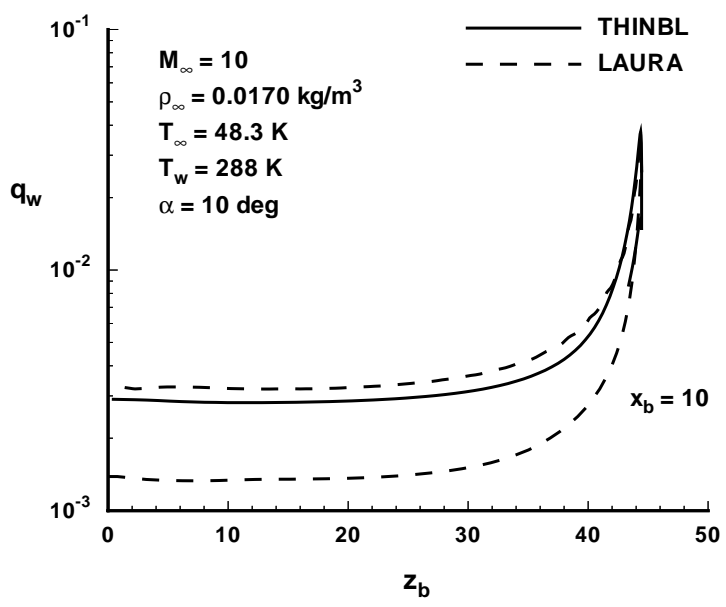

Figure 17. Circumferential heat transfer comparison for slender 3-D forebody.

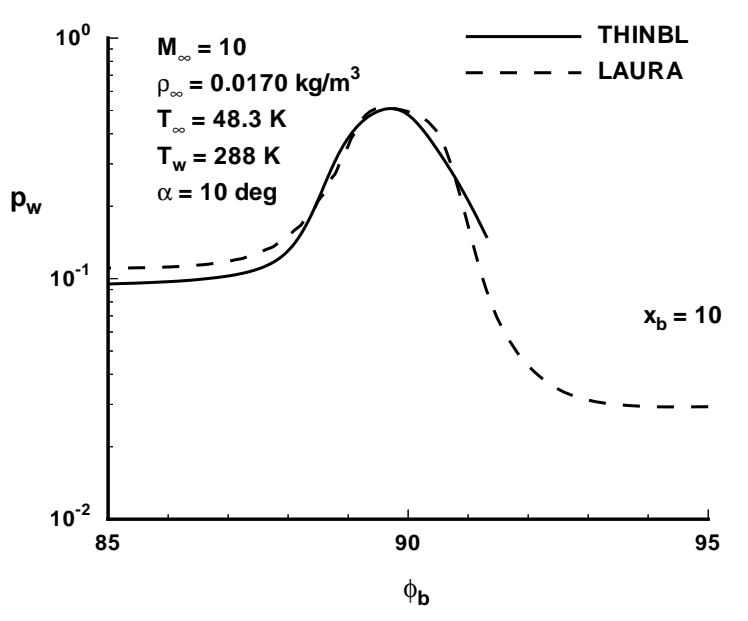

Figure 18. Circumferential pressure comparison for slender 3-D forebody.

and $z_{b}$ progresses toward the outermost point on the vehicle surface. The pressures and heating rates are essentially constant on the lower surface of the vehicle and increase dramatically as the edge is approached. Good agreement between the THINBL and LAURA solutions is seen on the flat lower surface and near the outer edge of the vehicle. A closer examination of the properties near the vehicle's edge is given in Figs. 18 and 19. The pressures and heating rates are presented with respect to $\phi_{b}$ with $\phi_{b}=90$ representing the edge of the vehicle. Good agreement (within 15 percent) between the THINBL and LAURA pressures is seen in this region. An interesting feature is noted in the heating rate comparisons in Fig. 19. Both THINBL and LAURA predict two peaks in the heating in this area. The peaks probably result from the fact that the super-ellipse cross-section of the vehicle is flat at $\phi_{b}=90$. The maximum curvature of the body crosssection is found at $\phi_{b}<90$ and $\phi_{b}>90$ (see Fig. 11). The surface streamlines diverge rapidly from these regions of

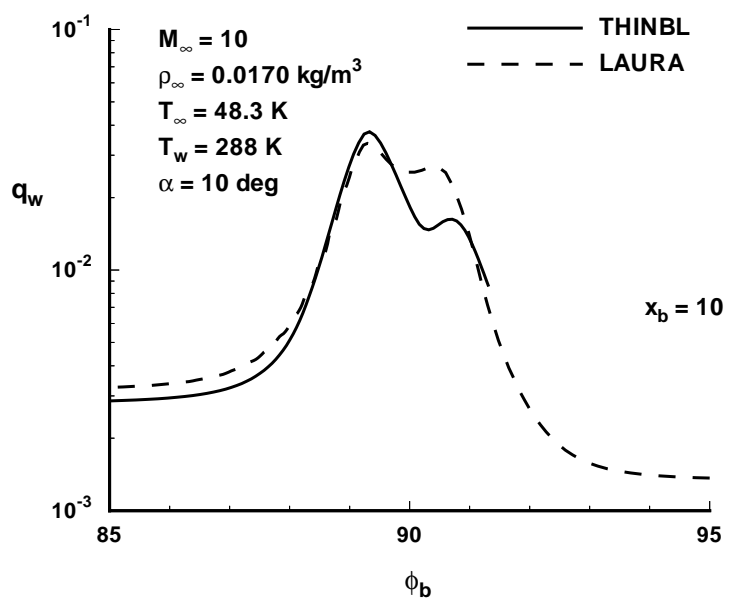

Figure 19. Circumferential heat transfer comparison for slender 3-D forebody.

high curvature. This streamline divergence may locally increase the heating levels. The magnitude of the heating rates from THINBL compare favorably with the LAURA solution for the first (and largest) peak, but underpredict the heating just on the leeside $\left(\phi_{b}>90\right)$. This ability to compute flowfield information and surface heating rates away from the plane of symmetry for a $3-D$ vehicle increases THINBL's applicability as a preliminary design tool.

\section{Acknowledgements}

The author wishes to thank Mr. Richard A. Thompson of the Aerothermodynamics Branch for providing the LAURA solution over the slender 3-D configuration at angle of attack. His assistance was invaluable and is greatly appreciated.

\section{Concluding Remarks}

An engineering inviscid-boundary layer method has been modified for application to hypersonic, slender 3-D vehicle forebodies. A new description of the shock shape in the nose region has been included. The engineering method with the new shock is applied to the solution over an axisymmetric power-law shape and a slender 3-D configuration at angle of attack. Comparisons with thin layer NS solutions demonstrate the accuracy of the new shock shape and its improvement over the original version. Surface pressures and heating rates compare favorably with the NS solution on the centerline and on the lower surface of the vehicle. The underprediction of the pressures and heating rates on the centerline aft of the nose should improve farther downstream. These modifications represent a significant improvement over current engineering methods and enhance current approximate 3-D capabilities for realistic slender geometries. 


\section{References}

${ }^{1}$ Riley, C. J. and DeJarnette, F. R., "Engineering Aerodynamic Heating Method for Hypersonic Flow," Journal of Spacecraft and Rockets, Vol. 29, May-June 1992, pp. 327-334.

${ }^{2}$ Riley, C. J., An Engineering Method for Interactive Inviscid-Boundary Layers in Three-Dimen sional Hypersonic Flow, PhD thesis, North Carolina State University, May 1992.

${ }^{3}$ Riley, C. J. and DeJarnette, F. R., "An Approximate Method for Calculating Three-Dimensional Inviscid Hypersonic Flow Fields," NASA TP-3018, Aug. 1990.

${ }^{4}$ Riley, C. J. and DeJarnette, F. R., "Engineering Calculations of Three-Dimensional Inviscid Hypersonic Flow Fields," Journal of Spacecraft and Rockets, Vol. 28, Nov.-Dec. 1991, pp. 628-635.

${ }^{5}$ Cooke, J. C., "An Axially Symmetric Analogue for General Three-Dimensional Boundary Layers," Aeronautical Research Council TR R\&M 3200, British Ministry of Aviation, June 1961.

${ }^{6}$ Zoby, E. V., Moss, J. N., and Sutton, K., “Approximate Convective-Heating Equations for Hypersonic Flows," Journal of Spacecraft and Rockets, Vol. 18, Jan.Feb. 1981, pp. 64-70.

${ }^{7}$ Maslen, S. H., "Inviscid Hypersonic Flow Past Smooth Symmetric Bodies," AIA A Journal, Vol. 2, June 1964, pp. $1055-1061$.

${ }^{8}$ Maslen, S. H., "Asymmetric Hypersonic Flow," NASA CR-2123, Sept. 1972.

${ }^{9}$ DeJarnette, F. R. and Hamilton, H. H., "Inviscid Surface Streamlines and Heat Transfer on Shuttle-Type Configurations," Journal of Spacecraft and Rockets, Vol. 10, May 1973, pp. 314-321.

${ }^{10}$ Hamilton, H. H., DeJarnette, F. R., and Weilmuenster, K. J., "Application of Axisymmetric Analog for Calculating Heating in Three-Dimensional Flows," Journal of Spacecraft and Rockets, Vol. 24, July-Aug. 1987, pp. 296-302.

${ }^{11}$ Maslen, S. H., "Development of a Method of Analysis and Computer Program for Calculating the Inviscid Flow About the Windward Surfaces of Space Shuttle Configurations at Large Angles of Attack," NASA CR132453, Jan. 1974.

${ }^{12}$ Cheatwood, F. M. and DeJarnette, F. R., "An Interactive User-Friendly Approach to Surface-Fitting ThreeDimensional Geometries," NASA CR-4126, March 1988.

${ }^{13}$ Hamilton, H. H., Greene, F. A., and DeJarnette, F. R., "An Approximate Method for Calculating Heating Rates on Three- Dimensional Vehicles," AIAA 93-2881, July 1993 .

${ }^{14}$ Gnoffo, P. A., "An Upwind-Biased, Point-Implicit Relaxation Algorithm for Viscous, Compressible PerfectGas Flows," NASA TP-2953, Feb. 1990. 